\title{
Einleitende Bemerkungen zur Gattungs- und Werksgeschichte des östlichen Manichäismus
}

\author{
JENS WILKENS (Göttingen)
}

Wenn wir vom östlichen Manichäismus sprechen, so beziehen wir uns auf den zentral- und ostasiatischen Manichäismus mit seiner Vielzahl von Quellensprachen: da sind zunächst die mitteliranischen Idiome, von denen zwei, die westmitteliranischen Sprachen Mittelpersisch und Parthisch, von den Manichäern Zentralasiens als prestigereiche Kirchensprachen verwendet wurden. Von besonderer Bedeutung ist das ostmitteliranische Sogdische - ursprünglich in der Gegend um Samarkand beheimatet, wurde es in den Kolonien sogdischer Händler und Kaufleute in weiträumigen Gebieten Zentralasiens und Chinas als Schrift- und Verkehrssprache gebraucht. Ein einzigartiger Text, der erst im Jahr 2009 zum ersten Mal vollständig publiziert wurde, ${ }^{1}$ ist ein kleinformatiges Fragment in manichäischer Schrift und baktrischer Sprache (M 1224). Es ist schon seit 1958 bekannt, aber erst die durch Textfunde der 1990er Jahre vertiefte Kenntnis des vor allem in Afghanistan beheimateten und eigentlich in griechischen Lettern geschriebenen ostmitteliranischen Baktrischen hat eine Deutung des Textes ermöglicht. Zusätzlich zu den vier genannten mitteliranischen Sprachen gibt es auch einige wenige frühneupersische Texte aus den Turfanfunden. Eine weitere wichtige Quellengruppe stellen die altuigurischen Texte dar, von denen ein großer Teil wohl aus mitteliranischen Sprachen übersetzt sein dürfte. Wir verfügen zwar nur über wenige eindeutig im Mitteliranischen und Altuigurischen überlieferte Paralleltexte, doch zeigt in vielen Fällen die Syntax der altuigurischen Quellen, dass es sich um Übersetzungen handeln muss. Die altuigurischen Originalwerke weisen z. T. ein formales Merkmal auf, das für die Dichtung der so genannten altaischen Völker charakteristisch ist: die Zeilenalliteration, besser bekannt unter der Bezeichnung ,altaischer Stabreim“.

Wir können davon ausgehen, dass die manichäische Gemeinde der Turfanoase zweisprachig (sogdisch-türkisch) war. Es kann als gesichert gelten, dass Manichäer, deren erste Sprache Altuigurisch war, auch Sogdisch geschrieben haben. ${ }^{2}$ Personennamen der Manichäer Zentralasiens sind nicht selten

1 Siehe Sims-Williams 2009.

2 Für den umgekehrten Fall, dass vermutlich ein Sogder einen altuigurischen Text abgeschrieben hat, siehe den Beitrag von KLAUS RÖHRBORN in diesem Band. 
hybrid, also aus iranischen und türkischen Bestandteilen zusammengesetzt. ${ }^{3}$ Die Begrifflichkeit der klerikalen Hierarchie ist aber durchweg iranisch. Ein Unikum stellt eine Bilingue in Tocharisch B (Westtocharisch) und Altuigurisch dar, die noch viele Rätsel aufgibt, z. B. ob wir auf der Grundlage dieser einen Handschrift eine manichäische Gemeinde von Sprechern des Westtocharischen postulieren dürfen. ${ }^{4}$ Zum östlichen Manichäismus rechnet man auch die chinesischen Texte, wobei in der Hauptsache drei umfangreiche Originaltexte bekannt sind, die alle in der einen oder anderen Weise eine Beeinflussung durch den iranischen Manichäismus verraten. Einige wenige Texte liegen in gleich mehreren östlichen Quellensprachen vor, so dass in solchen Fällen die komplexe Überlieferungsgeschichte nachvollzogen werden kann.

Der östliche Manichäismus wird in allgemeinen religionsgeschichtlichen Darstellungen dieser Religion häufig nur am Rande behandelt; dagegen wird den koptischen Quellen und antimanichäischen Berichten der Kirchenväter, also den westlichen Zeugnissen, deutlich mehr Beachtung geschenkt. Dabei ist gerade die manichäische Literatur aus dem Osten der Diaspora ausgesprochen reich und viele Werke finden sich nur hier. Dies gilt auch für Manis eigene Schriften, etwa das Šäbuhragān und das Gigantenbuch, die nur in der östlichen Überlieferung bezeugt sind. ${ }^{5}$

Die ersten manichäischen Originalzeugnisse überhaupt - es handelt sich um mitteliranische Fragmente aus den Funden der ersten deutschen Turfanexpedition (1902-1903) - wurden 1904 von F.W.K. MÜLLER der gelehrten Öffentlichkeit vorgestellt. ${ }^{6}$ So steht am Beginn der Erforschung der Originalquellen der Religion des Lichts die Auswertung iranischer Zeugnisse, bald gefolgt von Editionen chinesischer und altuigurischer Texte.

Der uigurische Manichäismus und seine literarischen Hinterlassenschaften finden meist noch weniger Beachtung als der iranische, dabei ist die Annahme des Manichäismus bei den Uiguren in den frühen 60er Jahren des 8. Jh. ein ganz singuläres Ereignis der manichäischen Religionsgeschichte. $\mathrm{Zu}$ den möglichen Gründen für die Konversion, die in verschiedenen Quellen behandelt wird, sind unterschiedliche Hypothesen entwickelt worden. Historisch bezeugt ist die Beteiligung sogdischer Missionare an der Bekehrung Bögü Kagans. Die Umstände dieses Ereignisses zeigen, dass hier bereits eine kulturelle Konstante vorgeprägt ist, ${ }^{7}$ die bis zum Niedergang des Manichäismus, der für Zentralasien vermutlich in das 11. Jh. zu datieren ist, Gültigkeit behalten sollte: die sogdisch-türkische Symbiose. Kontakte zwischen Sogdern und Türken reichen

3 Siehe ZIEME 2006.

4 Die Sammelhandschrift, zu der diese Bilingue gehört, ist Gegenstand des Beitrags von MiCHAEL KNÜPPEL in diesem Band.

5 Stoffe des Gigantenbuchs, die eventuell Auszüge aus diesem Werk darstellen, finden sich in der koptischen Literatur.

6 MÜller 1904a, 1904b.

7 Vgl. vor allem die wichtige Arbeit von Clark (2000). 
bekanntlich bis ins 6. Jh. zurück, als das Sogdische, das über viele Jahrhunderte als lingua franca an der nördlichen Seidenstraße und auch in den angrenzenden Steppenregionen fungierte, Kanzlei- und Schriftsprache im ersten türkischen Großreich war.

Während der Manichäismus in der Regel als verfolgte Religion ein Leben im Untergrund fristen musste, konnte er sich im Ostuigurischen Kaganat und im Westuigurischen Königreich unter staatlicher Patronage frei entfalten. Vor allem in der Turfanoase blühten manichäische Kunst und Literatur. Hier wurden manichäische Schriften gesammelt, vervielfältigt, übersetzt und illustriert; hier wurden ihm von staatlicher Seite Privilegien eingeräumt; hier konnte er seinen Einfluss auch auf politischer Ebene geltend machen. ${ }^{8}$ Briefe, Dokumente und Kolophone ${ }^{9}$ aus den Turfanfunden vermitteln Einblicke in das tägliche Leben der manichäischen Gemeinde des Westuigurischen Königreichs. Indem man Texte ins Altuigurische übertrug, entwickelte sich zum ersten Mal eine gepflegte türkische Literatursprache.

Da bis zum Untergang des Ostuigurischen Kaganates mit seinem Zentrum in der heutigen Mongolei die Uiguren die politischen Geschicke in den innerasiatischen Steppengebieten mitbestimmten und gar durch ihr Eingreifen den Untergang der Tang-Dynastie während der An Lushan-Rebellion verhinderten, wurde durch ihren Einfluss die Aktivität manichäischer Missionare in China geduldet. Wie in der Forschung schon mehrfach thematisiert, fallen der durch die Kirgisen herbeigeführte Zusammenbruch des Ostuigurischen Kaganats und die Verfolgung der Fremdreligionen im Reich der Mitte nicht von ungefähr zeitlich zusammen. ${ }^{10} \mathrm{Nach}$ der Katastrophe von 840 siedelte sich ein Teil der Uiguren in der Gegend der Turfanoase an, wo das Westuigurische Königreich mit seinen Hauptstädten Bešbalık (Beiting 北庭) und Kočo (Gaochang 高昌) gegründet wurde.

Vorliegender Band dokumentiert verschiedene Aspekte der Gattungs- und Werksgeschichte der Literatur des östlichen Manichäismus. Immer wieder scheint in den Beiträgen die enge Verflochtenheit der iranischen und türkischen Überlieferung auf. Es werden sowohl seit langem bekannte Texte und Gattungen unter neuen Fragestellungen untersucht als auch neue Quellen in Edition und Übersetzung vorgestellt. Das Buch gliedert sich in drei Teile: der erste widmet sich dem eigentlichen Thema des Symposions: der Gattungsgeschichte des manichäischen Schrifttums, der zweite umfasst eher textbezogene Arbeiten zur Werksgeschichte, während der dritte die chinesischen Forschungen zum Manichäismus zum Thema hat und bibliographische Informationen bietet.

\footnotetext{
8 KLimkeit 1982.

9 Siehe ZIEME 1992.

10 Vgl. etwa Lieu 1998, S. 128-131.
} 
Jens Wilkens

\section{Teil I: Gattungsgeschichte}

Neben christlichen Gleichnissen, die auch in den westlichen manichäischen Quellen bezeugt sind, haben die Manichäer Zentralasiens buddhistische Erzählungen adaptiert und vermutlich auch das Vorbild buddhistischer Jātaka- und Avadānasammlungen übernommen. ${ }^{11}$ Für die Erforschung der Adaption narrativer Stoffe ist die ostmanichäische Überlieferung besonders ergiebig. IRIS COLDITZ macht in ihrem Beitrag „Parabeln und Parabelabstrakta - Beobachtungen zu gattungsübergreifenden Lehr- und Erzählmotiven in den iranischen Manichaica" darauf aufmerksam, dass gewisse Lehr- und Erzählmotive in Texten verschiedener literarischer Genres vorkommen können. Kurzfassungen von Erzählungen und Parabeln können auch gattungsübergreifend eingesetzt werden - etwa im Kontext didaktischer oder liturgischer Werke. Die Autorin zeigt anhand von vier ausgewählten Beispielen aus der mittelpersischen, parthischen und sogdischen Literatur, in welcher Beziehung diese Kurzversionen zu den Langversionen stehen. Zudem wird eine Typologie der vorgestellten $\mathrm{Pa}-$ rabelabstrakta entwickelt und eine Hypothese zur Funktion dieser Textgattung vorgetragen.

Die Geschichte der mitteliranischen manichäischen Texte beginnt mit Mani selbst, der sein Šäbuhragān auf Mittelpersisch schrieb und es dem König der Sassaniden zueignete. DESMOND DUR KIN-MEISTERERNST stellt in „Literarische Termini in mitteliranischen manichäischen Texten“ diejenigen Begriffe zusammen, die sowohl einzelne Werke und Gattungen als auch Textteile oder -abschnitte (Refrain, Strophe) bezeichnen. Er behandelt die Bezeichnungen für Manis eigene Werke, für die aus den Turfanfunden reich bezeugte Hymnenliteratur, an der in der letzten Zeit besonders intensiv geforscht wurde, ${ }^{12}$ sowie für die Briefe, Lehrtraktate, Erzählungen, Parabeln usw.

Mehrfach sind schon sprachliche Differenzen in den buddhistischen, christlichen und manichäischen sogdischen Texten thematisiert worden. ANTJE WENDTLAND geht in ihrem Aufsatz „Orthographische und grammatische Besonderheiten manichäisch-soghdischer Texte und ihre Bedeutung für die Gattungen" speziell der statistisch dokumentierten Verteilung des so genannten maskulinen ( $x w)$ und femininen Artikels $\left(x^{\prime}\right)$ bei maskulinen und femininen Bezugswörtern nach. Die manichäischen Belege werden kontrastiv zu den buddhistischen und christlichen untersucht. Die Verfasserin kann zeigen, dass im Gegensatz zu den Grammatiken die Unterscheidung $x w$ vs. $x$ ' funktional bedingt und weniger auf das Genus des Bezugswortes bezogen ist. Ferner widmet sie sich dem unterschiedlichen Gebrauch von Konjunktionen und

11 Sundermann 1973, S. 5. Die Frage, ob diese Hypothese sich verifizieren lässt, kann erst ein formaler Vergleich manichäischer und buddhistischer Erzählungssammlungen klären.

12 Vgl. insbesondere die Editionen RECK 2004, DURKIN-MEISTERERNST 2006 und DURKIN-MEISTERERNST/MORANO 2010. 
dem Gebrauch von Imperfekt und Perfekt. Einige manichäische Texte verwenden regelmäßig das Perfekt im Erzähltext, während die buddhistischen Texte ursprünglich das Perfekt nur in der direkten Rede gebrauchen und dagegen das Imperfekt als Erzähltempus kennen. Eine weitere Gruppe manichäischer Texte gebraucht Imperfekt und Perfekt promiscue, während eine andere überwiegend das Perfekt verwendet. WENDTLAND macht hierfür unterschiedliche Textcorpora bzw. -gattungen verantwortlich.

Die religionsgeschichtliche Bedeutung der Turfanfunde auch über den zentralasiatischen Raum hinaus lässt sich sehr gut am Beispiel der ostmanichäischen Überlieferung von Manis Gigantenbuch demonstrieren. Die mitteliranischen und altuigurischen Textzeugnisse zeigen sehr enge Parallelen zu dem zur Henoch-Literatur gehörigen Gigantenbuch, das in mehreren Abschriften aus den Qumranfunden bekannt ist. JENS WILKENS vergleicht in „Funktion und gattungsgeschichtliche Bedeutung des manichäischen Gigantenbuchs" die Mythologeme des jüdischen Werkes, wie sie größtenteils auch von Mani übernommen wurden, mit Manis eigenem mythologischen System und zeigt, an welchen Punkten sich Widersprüche und gegenläufige Tendenzen ergeben. Indem Mani dem eigentlichen Text eine in ihrem dogmatischen Gehalt typisch manichäische Deutung nachschaltet, werden die in der Frühzeit des Menschengeschlechts angesiedelten Ereignisse des Gigantenbuchs, wie sie auch das aramäische Werk aus Qumran kennt, psychologisch-anthropologisch ausgelegt. Mani ist nicht der einzige Autor der Spätantike, der diesen Weg der Exegese eingeschlagen hat.

\section{Teil II: Werksgeschichte}

MiCHAEL KNÜPPELs Beitrag „Zur späten manichäisch-uigurischen Dichtung“ ist in engerem Sinne der Datierung einer Sammelhandschrift gewidmet, berührt aber gleichzeitig einen größeren Themenkomplex: die kontrovers diskutierte Frage, über welchen Zeitraum sich die altuigurische manichäische Literatur erstreckt und hier vor allem, wann sie unterging. Der Verfasser spricht sich nach einer Prüfung der von unterschiedlichen Gelehrten vorgetragenen Argumente für eine Frühdatierung selbst für eine späte Datierung (13. Jh.) dieses einzigen manichäischen Manuskripts im indischen Pustaka-Format aus. Einzigartig ist die Handschrift auch deshalb, weil sie die erwähnte Bilingue Tocharisch B/Altuigurisch enthält.

ENRICO MORANO widmet sich in „New Research on Mani’s Book of Giants" dem bereits genannten Werk des Religionsstifters. Anschließend an seine Übersetzung aller bekannten Gigantenbuchfragmente und -auszüge ins Italienische gibt er neben einer Aufzählung der dramatis personae auch eine in Teilen hypothetische Abfolge der Handlungsstränge, wobei er jeder der zwölf von ihm identifizierten 'Sequenzen' die jeweiligen Manuskriptzeugen zuordnet. Da sowohl das aramäische als auch das in mehreren Sprachen bezeugte manichäi- 
sche Gigantenbuch nur fragmentarisch erhalten sind, ist dies ein willkommener Versuch, die Fragmente erstmalig in eine sinnhafte Ordnung zu bringen. Auch Parallelen in der biblischen und pseudepigraphischen Literatur werden verzeichnet. Da das aramäische Gigantenbuch innerhalb des Corpus der HenochLiteratur eine Sonderstellung innehat, ist die Rekonstruktion des Handlungsverlaufs besonders schwierig. Eine Zugabe ist die Transliteration der Rückseite des sogdischen Gigantenbuchfragments M 500n, die erst durch eine kürzlich vorgenommene Restaurierung freigelegt werden konnte.

Der vermutlich bekannteste altuigurische manichäische Text ist das Xuāstvānift. Es liegt in zahlreichen Abschriften vor, die ganz unterschiedliche Formate besitzen, von sehr kleinen Kodices bis hin zu langen Buchrollen. Eine offensichtliche Gliederung in 15 Abschnitte gibt der Text selbst durch Setzung von Ordinalzahlen vor. In seiner Bearbeitung hatte WILLI BANG eine weitere Unterteilung der einzelnen Abschnitte vorgenommen. ${ }^{13}$ ZEKINE ÖZERTURAL, deren Neuedition dieses bedeutenden Sündenbekenntnisses vor dem Abschluss steht, setzt sich in ihrem Beitrag ,Die innere Gliederung des alttürkischen Beichttextes Xuāstvānīft" kritisch mit BANGs Einteilung auseinander und schlägt eine neue Untergliederung einzelner Abschnitte ('Kapitel') vor. Ferner hat sie erstmalig erkannt, dass das Xuāstvānīft in zwei Hauptteile zerfällt, so dass sich die Abschnitte 1-7 und 8-14 zusammenfassen lassen, während der Punkt 15 als eine Art Zusammenfassung für sich steht.

Weniger der Gattungs- und Werksgeschichte der manichäischen Literatur als vielmehr einem Problem der Kosmologie und Mythologie ist der Aufsatz von ANTONIO PANAINO ,Sadwēs, Anāhīd and the Manichaean Maiden of Light" gewidmet: der Funktion und mythologischen Bedeutung der weiblichen Funktionsgestalt, die im Parthischen mit dem Namen Sadwēs bezeichnet wird und in der avestischen Literatur als Name eines Sterns (Satauuaēsa) belegt ist. Der Verfasser schildert die Rolle von Satauuaēsa im Rahmen der altiranischen Mythologie und Astrologie, wo Satauuaēsa eine männliche Gottheit ist, sowie von Sadwēs in der Pahlavi-Literatur und vergleicht diese mit der von Sadwēs bzw. der dieser gleichgesetzten Jungfrau des Lichts im manichäischen System. Hier ist Sadwēs kein eigentlicher Stern mehr, operiert aber in der himmlischen Sphäre bei der für den manichäischen Mythos konstitutiven Episode von der Verführung der Archonten.

Es ist schon früh bemerkt worden, dass unter den in der Turfanoase geborgenen Handschriftenresten Sammelhandschriften identifiziert werden können, die nicht nur Werke aus unterschiedlichen Genres der manichäischen Literatur enthalten, sondern auch in verschiedenen Sprachen abgefasst sind. CHRISTIANE RECK legt in „Fragmente von Büchern - Zwei Sammelhandschriften im Vergleich" erstmalig eine ausführliche inhaltliche und strukturelle Analyse zweier Sammelhandschriften vor. Zur ersten Sammelhandschrift in sogdouigurischer Schrift gehören Texte in sogdischer und altuigurischer Sprache

13 Vgl. BANG 1923. 
sowie Reste von parthischen und mittelpersischen Hymnen, wobei auch Zwischenüberschriften in türkischer Runenschrift, Glossen in manichäischer Schrift sowie baktrische Buchstaben vorkommen. Die zweite Sammelhandschrift ist in manichäischer Schrift geschrieben und enthält parthische Hymnenzyklen und das altuigurische Xuāstvānīft. Nach einer Vorstellung aller enthaltenen Texte werden Hypothesen zur Funktion und möglichen liturgischen Verwendung dieser Sammelhandschriften entwickelt. Hier wird der Themenbereich „Gattungsgeschichte“ notwendigerweise ebenfalls berührt. Unveröffentlichte Stücke werden in Transliteration und Übersetzung publiziert.

KLAUS RÖHRBORN untersucht in ,Zum Schrifttum der westtürkischen Manichäer" den Schreiberkolophon der altuigurischen Prachthandschrift MIK III 198, in dem der Herrscher El-tirgök Alp Burgučan Alp Tarkan Bäg erwähnt wird. Er geht der Frage nach, warum ein im Westen, im Čigil-Reich, residierender Fürst in einem Kolophon genannt wird, der in der Turfan-Oase gefunden wurde, und diskutiert frühere Erklärungsversuche. Er kommt zu dem Ergebnis, dass das Manuskript aus dem Siebenstromland stammen muss und von Manichäern aus dieser Region nach Kočo gebracht wurde. Wie vor ihm schon LIVŠIC schreibt er den Text der sogdisch-türkischen Symbiose zu und vermutet, dass der Schreiber und der Verfasser des Kolophon-Textes ein Sogder aus dem Siebenstromland war. Die Leserkolophone seien dann von späteren Benutzern in der Turfan-Oase hinzugefügt worden. Werksgeschichte ist hier also vor allen Dingen die Feststellung der Provenienz des Manuskripts, die ein Schlaglicht auf bislang nicht oder nur kaum erforschte historische Vorgänge nach dem Zusammenbruch des Ostuigurischen Kaganats nach dem Jahr 840 wirft.

Der im Jahr 1969 in Ägypten aufgefundene Kölner Mani-Kodex, das kleinste Buch der Antike, hat nicht nur bis dahin unbekannte bzw. umstrittene Details aus der Biographie des Religionsstifters erhellt, sondern auch dessen Selbstverständnis als Lichtapostel mit der Person Pauli verknüpft. Auch wenn Paulus als Name in den zentralasiatischen Manichaica durchaus belegt ist, die Bedeutung, die ihm der griechisch-sprachige Kölner Mani-Kodex zuweist, wäre den östlichen Quellen kaum zu entnehmen gewesen. ALOÏs VAN TONGERLOO gibt in „Einige Notizen zur Stellung Pauli im Kölner Mani-Kodex“ eine Übersicht zu dieser Thematik. Es handelt sich um die einzige Studie des vorliegenden Bandes zu einer westlichen manichäischen Quelle. Der Verfasser kann zeigen, dass die Berufung Pauli als Vorbild für die literarische Verarbeitung von Manis Berufung diente, die zu den zentralen Themen des Kölner Mani-Kodexes zählt.

Von entscheidender Bedeutung für die manichäische Literatur - insbesondere für die Homilien - waren die Ereignisse, seien sie nun historisch verbürgt oder hagiografisch gefärbt, die mit dem Leben des Religionsstifters in Verbindung standen. Eine ziemlich umfangreiche, wenn auch fragmentarische mitteliranische Materialbasis steht der Forschung vor allem durch die Arbeiten 
WERNER SUNDERMANNs zur Verfügung. ${ }^{14}$ Altuigurische Versionen kirchengeschichtlicher Texte, die auf Ereignisse des 3. Jh. n. Chr. Bezug nehmen, sind relativ selten. Von hohem Interesse ist daher der Aufsatz von PETER ZIEME „Zwei altuigurische Bekehrungstexte aus der manichäischen Missionsgeschichte", in dem er weitere altuigurische Materialien ediert. Der erste Text von der Bekehrung des Mihršāh in einem stark sogdisch anmutenden Duktus hat eine parthische Parallele. Ob es sich bei dem zweiten Text um eine Bekehrungsgeschichte handelt ist nicht ganz sicher.

\section{Teil III: Der östliche Manichäismus in der chinesischen Forschung}

Durch die vorbildliche Bibliographie von GUNNER MIKKELSEN lässt sich die Forschungsgeschichte der manichäischen Studien sehr gut verfolgen. ${ }^{15}$ Sie enthält auch viele chinesisch-sprachige Titel, doch sind gerade in den letzten Jahren viele Arbeiten zum Manichäismus in China erschienen. ERKIN ARIZ legt in „Bibliographie der in der Volksrepublik China verfassten Arbeiten über den Manichäismus" eine bibliographische Zusammenschau in chronologischer Anordnung vor, die bis in die Gegenwart reicht. Es werden auch neuuigurische Titel berücksichtigt.

ABLET SEMET skizziert in „Die chinesischen Forschungen zum iranischen und zum uigurischen Manichäismus“ die Entwicklungslinien der auf den Raum Zentralasien bezogenen Manichäologie in China und gibt eine thematisch geordnete Übersicht über die wichtigsten Publikationen auf diesem Gebiet. Gerade die neueren Arbeiten sind der westlichen Forschung bislang weitgehend verschlossen geblieben, so dass man sich anhand des Aufsatzes über den Forschungsstand informieren kann.

\section{Bibliographie}

BANG, Willi (1923): Manichaeische Laien-Beichtspiegel. In: Le Muséon 36, S. 137242.

ClARK, LARRY (2000): The Conversion of Bügü Khan to Manichaeism. In: RONALD E. Emmerick / Werner Sundermann / Peter Zieme (Hrsg.), Studia Manichaica. IV. Internationaler Kongreß zum Manichäismus, Berlin, 14.-18. Juli 1997, Berlin (Berichte und Abhandlungen / Berlin-Brandenburgische Akademie der Wissenschaften; Sonderband 4), S. 83-123.

KLIMKEIT, HANS-JOACHIM (1982): Manichaean Kingship: Gnosis at Home in the World. In: Numen 29, 17-32.

14 Siehe insbesondere die Edition SUNDERMANN 1981.

15 MiKKELSEN 1997. 
Durkin-Meisterernst, Desmond (2006): The Hymns to the Living Soul. Middle Persian and Parthian Texts in the Turfan Collection, Turnhout (Berliner Turfantexte XXIV).

Durkin-Meisterernst, Desmond / Enrico Morano (2010): Mani's Psalms. Middle Persian, Parthian and Sogdian fragments in the Turfan Collection, Turnhout (Berliner Turfantexte XXVII).

Lieu, Samuel N. C. (1998): Manichaeism in Central Asia \& China, Leiden/Boston/Köln (Nag Hammadi and Manichaean Studies XLV).

Mikkelsen, Gunner (1997): Bibliographia Manichaica. A Comprehensive Bibliography of Manichaeism through 1996, Turnhout (Corpus Fontium Manichaeorum; Subsidia I).

Müller, F[RIEDRICH] W[ILHELM] K[ARL] (1904a): Handschriften-Reste in EstrangeloSchrift aus Turfan, Chinesisch-Turkistan [I], Berlin (aus: Sitzungsberichte der Preußischen Akademie der Wissenschaften, Phil.-hist. Klasse, S. 348-352).

MÜller, F[RIEDRICH] W[ILHELM] K[ARL] (1904b): Handschriften-Reste in EstrangeloSchrift aus Turfan, Chinesisch-Turkistan II. Teil, Berlin (Abhandlungen der Preußischen Akademie der Wissenschaften, Anhang, Nr. 2).

ReCK, Christiane (2004): Gesegnet sei dieser Tag: Manichäische Festtagshymnen. Edition der mittelpersischen und parthischen Sonntags-, Montags- und Bemahymnen, Turnhout (Berliner Turfantexte XXII).

Sims-Williams, NichOlas (2009): The Bactrian Fragment in Manichaean Script (M 1224). In: DeSMOND DURKIN-MEISTERERnst / CHRistiane RecK / DieTER WEBER (Hrsg.), Literarische Stoffe und ihre Gestaltung in mitteliranischer Zeit. Kolloquium anlässlich des 70. Geburtstages von Werner Sundermann, Wiesbaden (Beiträge zur Iranistik 31), S. 245-268.

SUNDERMANN, WERNER (1973): Mittelpersische und parthische kosmogonische und Parabeltexte der Manichäer, Berlin (Berliner Turfantexte IV).

SUNDERMANN, WERNER (1981): Mitteliranische manichäische Texte kirchengeschichtlichen Inhalts mit einem Appendix von NICHOLAS SIMS-WILLIAMS, Berlin (Berliner Turfantexte XI).

Zieme, Peter (1992): Manichäische Kolophone und Könige. In: GeRnOT Wiessner / Hans-JOACHIM Klimkeit (Hrsg.), Studia Manichaica II. Internationaler Kongreß zum Manichäismus. 6.-10. August 1989, St. Augustin/Bonn, Wiesbaden, S. 319-327.

Zieme, Peter (2006): Hybrid Names as a Special Device of Central Asian Naming. In: Lars Johanson / Christiane Bulut (Hrsg.), Turkic-Iranian Contact Areas. Historical and Linguistic Aspects, Wiesbaden (Turcologica 62), S. 114-127. 
